\title{
Front Matter: Volume 7589
}

, "Front Matter: Volume 7589," Proc. SPIE 7589, Frontiers in Ultrafast Optics: Biomedical, Scientific, and Industrial Applications X, 758901 (19 March 2010); doi: $10.1117 / 12.861797$

SPIE. Event: SPIE LASE, 2010, San Francisco, California, United States 


\title{
PROCEEDINGS OF SPIE
}

\section{Frontiers in Ultrafast Optics: Biomedical, Scientific, and Industrial Applications $X$}

\author{
Alexander Heisterkamp \\ Joseph Neev \\ Stefan Nolte \\ Rick P. Trebino \\ Editors \\ 24-26 January 2010 \\ San Francisco, California, United States \\ Sponsored by \\ SPIE \\ Cosponsored by \\ Amplitude Systèmes (France) \\ APE GmbH (Germany) \\ High Q Laser Production GmbH (Austria) \\ IMRA America, Inc. (United States) \\ Newport Spectra-Physics (United States) \\ Time-Bandwidth Products AG (Switzerland) \\ Published by \\ SPIE
}

Volume 7589 
The papers included in this volume were part of the technical conference cited on the cover and title page. Papers were selected and subject to review by the editors and conference program committee. Some conference presentations may not be available for publication. The papers published in these proceedings reflect the work and thoughts of the authors and are published herein as submitted. The publisher is not responsible for the validity of the information or for any outcomes resulting from reliance thereon.

Please use the following format to cite material from this book:

Author(s), "Title of Paper," in Frontiers in Ultrafast Optics: Biomedical, Scientific, and Industrial Applications $X$, edited by Alexander Heisterkamp, Joseph Neev, Stefan Nolte, Rick P. Trebino, Proceedings of SPIE Vol. 7589 (SPIE, Bellingham, WA, 2010) Article CID Number.

ISSN 0277-786X

ISBN 9780819479853

Published by

SPIE

P.O. Box 10, Bellingham, Washington 98227-0010 USA

Telephone +1 3606763290 (Pacific Time) · Fax +1 3606471445

SPIE.org

Copyright (@ 2010, Society of Photo-Optical Instrumentation Engineers

Copying of material in this book for internal or personal use, or for the internal or personal use of specific clients, beyond the fair use provisions granted by the U.S. Copyright Law is authorized by SPIE subject to payment of copying fees. The Transactional Reporting Service base fee for this volume is $\$ 18.00$ per article (or portion thereof), which should be paid directly to the Copyright Clearance Center (CCC), 222 Rosewood Drive, Danvers, MA 01923. Payment may also be made electronically through CCC Online at copyright.com. Other copying for republication, resale, advertising or promotion, or any form of systematic or multiple reproduction of any material in this book is prohibited except with permission in writing from the publisher. The CCC fee code is 0277-786X/10/\$18.00.

Printed in the United States of America.

Publication of record for individual papers is online in the SPIE Digital Library.

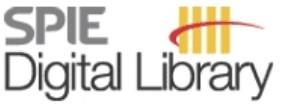

SPIEDigitalLibrary.org

Paper Numbering: Proceedings of SPIE follow an e-First publication model, with papers published first online and then in print and on CD-ROM. Papers are published as they are submitted and meet publication criteria. A unique, consistent, permanent citation identifier (CID) number is assigned to each article at the time of the first publication. Utilization of CIDs allows articles to be fully citable as soon they are published online, and connects the same identifier to all online, print, and electronic versions of the publication. SPIE uses a six-digit CID article numbering system in which:

- The first four digits correspond to the SPIE volume number.

- The last two digits indicate publication order within the volume using a Base 36 numbering system employing both numerals and letters. These two-number sets start with 00, 01, 02, 03, 04 , 05, 06, 07, 08, 09, OA, OB ... 0Z, followed by 10-1Z, 20-2Z, etc.

The CID number appears on each page of the manuscript. The complete citation is used on the first page, and an abbreviated version on subsequent pages. Numbers in the index correspond to the last two digits of the six-digit CID number. 


\section{Contents}

vii Conference Committee

ix Ultrafast fiber laser technology: status and prospects (Plenary Paper) [7579-102]

A. Tünnermann, J. Limpert, Friedrich-Schiller-Univ. Jena (Germany) and Fraunhofer Institute for Applied Optics and Precision Engineering (Germany)

\section{SPECIAL SESSION: ULTRAFAST LASERS IN OPHTHALMOLOGY}

758904 Femtosecond lasers in ophthalmology: clinical applications in anterior segment surgery (Invited Paper) [7589-01]

T. Juhasz, Univ. of California, Irvine (United States) and LensxLasers Inc. (United States):

Z. Nagy, Semmelweis Univ. (Hungary); M. Sarayba, LensxLasers Inc. (United States);

R. M. Kurtz, Univ. of California, Irvine (United States) and LensxLasers Inc. (United States)

758906 High resolution macroscopy (HRMac) of the eye using nonlinear optical imaging (Invited Paper) [7589-03]

M. Winkler, B. E. Jester, C. Nien-Shy, D. Chai, D. J. Brown, J. V. Jester, The Gavin Herbert Eye Institute, Univ. of California Irvine Medical Ctr. (United States)

\section{MULTIPHOTON IMAGING}

758907 Brain plasticity and functionality explored by nonlinear optical microscopy (Invited Paper) [7589-04]

L. Sacconi, L. Allegra, Univ. degli Studi di Firenze (Italy); M. Buffelli, Univ. degli Studi di Verona (Italy); P. Cesare, CERC Fondazione Santa Lucia (Italy); E. D'Angelo, D. Gandolfi, Univ. degli Studi di Pavia (Italy); G. Grasselli, CERC Fondazione Santa Lucia (Italy); J. Lotti, Univ. degli Studi di Firenze (Italy); J. Mapelli, Univ. degli Studi di Pavia (Italy); P. Strata, CERC Fondazione Santa Lucia (Italy); F. S. Pavone, Univ. degli Studi di Firenze (Italy)

758908 A novel flexible clinical multiphoton tomograph for early melanoma detection, skin analysis, testing of anti-age products, and in situ nanoparticle tracking [7589-05] M. Weinigel, H. G. Breunig, A. Gregory, P. Fischer, M. Kellner-Höfer, R. Bückle, JenLab GmbH (Germany); K. König, JenLab GmbH (Germany) and Saarland Univ. (Germany)

\section{fs LASERS FOR CELL MANIPULATION}

7589 OA Functional enucleation of porcine oocytes for somatic cell nuclear transfer using femtosecond laser pulses [7589-07]

K. Kuetemeyer, Laser Zentrum Hannover e.V. (Germany); A. Lucas-Hahn, B. Petersen, P. Hassel, E. Lemme, H. Niemann, Friedrich-Loeffler-Institute (Germany); A. Heisterkamp, Laser Zentrum Hannover e.V. (Germany) 
7589 OC Fs-laser cell perforation using gold nanoparticles of different shapes [7589-09]

M. Schomaker, H. Fehlaver, Laser Zentrum Hannover e.V. (Germany) and Research Cluster of Excellence REBIRTH (Germany); W. Bintig, A. Ngezahayo, Leibniz Univ. Hannover (Germany); I. Nolte, H. Murua Escobar, Research Cluster of Excellence REBIRTH, Univ. of Veterinary Medicine Hannover (Germany); H. Lubatschowski, A. Heisterkamp, Laser Zentrum Hannover e.V. (Germany) and Research Cluster of Excellence REBIRTH (Germany)

7589 OD Dual-beam optical trapping of cells in an optofluidic device fabricated by femtosecond lasers [7589-10]

N. Bellini, IFN-CNR, Politecnico di Milano (Italy); F. Braheri, Univ. degli Studi di Pavia (Italy); K. C. Vishnubhatla, IFN-CNR, Politecnico di Milano (Italy); L. Ferrara, P. Minzioni, Univ. degli Studi di Pavia (Italy); G. Cerullo, R. Ramponi, IFN-CNR, Politecnico di Milano (Italy); I. Cristiani, Univ. degli Studi di Pavia (Italy); R. Osellame, IFN-CNR, Politecnico di Milano (Italy)

\section{FEMTOSECOND LASERS AND COMPONENTS}

7589 Ol Applications of femtosecond laser induced Bragg gratings in silica and non-silica based optical fibers (Invited Paper) [7589-15]

S. J. Mihailov, D. Grobnic, C. W. Smelser, P. Lu, R. B. Walker, H. Ding, Communications Research Ctr. Canada (Canada)

7589 0J Mode selective fiber Bragg gratings [7589-16]

J. U. Thomas, C. Voigtländer, S. Nolte, Friedrich-Schiller-Univ. Jena (Germany);

A. Tünnermann, Friedrich-Schiller-Univ. Jena (Germany) and Fraunhofer Institute for Applied Optics and Precision Engineering (Germany); N. Jovanovic, G. D. Marshall, M. J. Withford, M. Steel, Macquarie Univ. (Australia)

7589 OK Compact 60fs multigigawatt diode-pumped laser using postcompression technique [7589-17]

A. Courjaud, Amplitude Systèmes (France); E. Mével, E. Constant, CELIA, CNRS, Univ. Bordeaux 1 (France); E. Mottay, Amplitude Systèmes (France)

$7589 \mathrm{OL}$ Compression of idler pulses with an identical positive dispersive media to signal pulse stretcher in ultrafast optical-parametric chirped-pulse amplification [7589-18] Y. Akahane, K. Ogawa, K. Tsuji, M. Aoyama, K. Yamakawa, Japan Atomic Energy Agency (Japan)

7589 OM Broadband $\mathrm{Yb}: \mathrm{CaF}_{2}$ regenerative amplifier for millijoule range ultrashort pulse amplification [7589-19]

S. Ricaud, M. Delaigue, A. Courjaud, Amplitude Systèmes (France); F. Druon, P. Georges, Lab. Charles Fabry de I'Institut d'Optique, CNRS, Univ. Paris-Sud 11 (France); P. Camy, J.-L. Doualan, R. Moncorgé, ENSICAEN, CNRS, Univ. de Caen (France); E. Mottay, Amplitude Systèmes (France)

\section{MODELING AND MEASUREMENT}

7589 ON Measuring complex and visible ultrashort pulses [7589-20]

D. Lee, Swamp Optics, LLC (United States); L. Xu, R. Trebino, Georgia Institute of Technology (United States) 
758900 Modeling the propagation of ultrashort pulses through optical systems [7589-21] F. Wyrowski, Friedrich-Schiller-Univ. Jena (Germany); C. Hellmann, R. Krieg, H. Schweitzer, LightTrans GmbH (Germany)

$7589 \mathrm{OQ}$ Surface-enhanced Raman scattering hot spot isolation using surface-enhanced multiphoton lithography (Best Student Paper Award) [7589-23]

E. D. Diebold, P. Peng, E. Mazur, Harvard Univ. (United States)

fs LASER-INDUCED WAVEGUIDES

7589 OT Curved-waveguide fabrication using femtosecond laser processing with glass hologram [7589-26]

J. Suzuki, Y. Arima, M. Yamaji, H. Kawashima, S. Tanaka, New Glass Forum (Japan)

7589 OU Direct femtosecond laser written waveguides in bulk $\mathrm{Ti}^{3+}$ :sapphire [7589-27]

S. Gross, M. J. Withford, A. Fuerbach, Macquarie Univ. (Australia)

7589 OV Soliton formation near topological defects in waveguide arrays [7589-28]

M. Heinrich, R. Keil, Friedrich-Schiller-Univ. Jena (Germany); A. Szameit, Technion-Israel Institute of Technology (Israel); F. Dreisow, Friedrich-Schiller-Univ. Jena (Germany); S. Nolte, A. Tünnermann, Friedrich-Schiller-Univ. Jena (Germany) and Fraunhofer Institute for Applied Optics and Precision Engineering (Germany)

ULTRASHORT PULSE MICROMACHINING: JOINT SESSION WITH CONFERENCE 7584

758913 Ultrafast laser-based tools enhance solar cell efficiencies [7589-37]

F. Colville, Coherent, Inc. (United States)

758914 Picosecond laser patterning of $\mathrm{NiCr}$ thin film strain gages [7589-38]

O. Suttmann, Laser Zentrum Hannover e.V. (Germany); M. Gosselin, The Univ. of British Columbia (Canada); U. Klug, R. Kling, Laser Zentrum Hannover e.V. (Germany)

758915 High repetition rate femtosecond laser processing of metals [7589-39]

J. Schille, Univ. of Applied Sciences Mittweida (Germany) and Univ. of Manchester (United Kingdom); R. Ebert, U. Loeschner, Univ. of Applied Sciences Mittweida (Germany); P. Scully, N. Goddard, Univ. of Manchester (United Kingdom); H. Exner, Univ. of Applied Sciences Mittweida (Germany)

FEMTOSECOND LASER NANOPROCESSING: JOINT SESSION WITH CONFERENCE 7584

758916 Brighter incandescent light sources from the black metal (Invited Paper) [7589-40]

A. Y. Vorobyev, C. Guo, The Institute of Optics, Univ. of Rochester (United States) 
THREE-DIMENSIONAL DIRECT WRITING: JOINT SESSION WITH CONFERENCE 7584

758919 Birefringent elements based on femtosecond laser-induced nanogratings [7589-43]

L. P. R. Ramirez, M. Heinrich, S. Richter, F. Dreisow, R. Keil, Friedrich-Schiller-Univ. Jena (Germany); A. V. Korovin, U. Peschel, Friedrich-Alexander-Univ. Erlangen-Nürnberg (Germany); S. Nolte, A. Tünnermann, Friedrich-Schiller-Univ. Jena (Germany) and Fraunhofer Institute for Applied Optics and Precision Engineering (Germany)

7589 1A Femtosecond laser written embedded diffractive optical elements and their applications [7589-44]

J. Choi, M. Ramme, T. Anderson, M. C. Richardson, CREOL, The College of Optics and Photonics, Univ. of Central Florida (United States)

\section{POSTER SESSION}

7589 1B Observations of the intense soft $x$-ray emissions from ultrathin Au films irradiated with high contrast laser pulses [7589-45]

M. Ishino, M. Kado, M. Nishikino, Japan Atomic Energy Agency (Japan); K. Shinohara, Waseda Univ. (Japan); S. Tamotsu, K. Yasuda, Nara Women's Univ. (Japan); N. Hasegawa, M. Kishimoto, T. Ohba, T. Kawachi, Japan Atomic Energy Agency (Japan)

$75891 \mathrm{~N} \quad$ Nonlinear lithographic properties by femtosecond laser pulses using a low-NA lens [7589-48]

M. Mizoshiri, H. Nishiyama, Osaka Univ. (Japan); J. Nishii, Hokkaido Univ. (Japan); Y. Hirata, Osaka Univ. (Japan)

7589 1G Laser microsintering of tungsten in vacuum [7589-50]

R. Ebert, F. Ullmann, L. Hartwig, T. Suess, S. Kloetzer, A. Streek, J. Schille, P. Regenfuss, H. Exner, Univ. of Applied Sciences Mittweida (Germany)

Author Index 


\title{
Conference Committee
}

\author{
Symposium Chairs
}

Donald J. Harter, IMRA America, Inc. (United States)

Peter R. Herman, University of Toronto (Canada)

Symposium Cochairs

Alberto Piqué, Naval Research Laboratory (United States)

Friedhelm Dorsch, TRUMPF Photonics (United States)

Conference Chairs

Alexander Heisterkamp, Laser Zentrum Hannover e.V. (Germany) Joseph Neev, Femto-Sec Tech, Inc. (United States)

Stefan Nolte, Friedrich-Schiller-Universität Jena (Germany)

Rick P. Trebino, Georgia Institute of Technology (United States)

Program Committee

Craig B. Arnold, Princeton University (United States)

Adela Ben-Yakar, The University of Texas at Austin (United States)

James E. Carey III, SiOnyx Inc. (United States)

Denise M. Krol, University of California, Davis (United States)

Holger Lubatschowski, Laser Zentrum Hannover e.V. (Germany)

Eric D. Mazur, Harvard University (United States)

Eric Mottay, Amplitude Systèmes (France)

Christopher B. Schaffer, Cornell University (United States)

Alexander Szameit, Technion-Israel Institute of Technology (Israel)

Alfred Vogel, Institute of Biomedical Optics, Universität zu Lübeck

(Germany)

Wataru Watanabe, National Institute of Advanced Industrial Science and Technology (Japan)

Session Chairs

1 Special Session: Ultrafast Lasers in Ophthalmology

Holger Lubatschowski, Laser Zentrum Hannover e.V. (Germany)

2 Multiphoton Imaging

Joseph Neev, FemtoSec Tech, Inc. (United States) 
3 fs Lasers for Cell Manipulation

Adela Ben-Yakar, The University of Texas at Austin (United States)

4 Applications of fs Lasers for Surgery and Biomedical Devices

Joseph Neev, Femto-Sec Tech, Inc. (United States)

$5 \quad$ Femtosecond Lasers and Components

James E. Carey III, SiOnyx Inc. (United States)

$6 \quad$ Modeling and Measurement

Rick P. Trebino, Georgia Institute of Technology (United States)

7 fs Laser-Induced Waveguides

Denise M. Krol, University of California, Davis (United States)

8 fs Laser Modification of Glass

Eric Mottay, Amplitude Systèmes (France)

$9 \quad$ Ultrashort Pulse Micromachining: Joint Session with Conference 7584

Michel Meunier, Ecole Polytechnique de Montréal (Canada)

10 Femtosecond Laser Nanoprocessing: Joint Session with

Conference 7584

Guido Hennig, MDC Max Daetwyler AG (Switzerland)

$11 \quad$ Nonlinear Processing: Joint Session with Conference 7584

Wataru Watanabe, National Institute of Advanced Industrial Science and Technology (Japan)

12 Three-Dimensional Direct Writing: Joint Session with Conference 7584

Stefan Nolte, Friedrich-Schiller-Universität Jena (Germany) 